\title{
Wonderworld of the microbiome
}

\author{
Adrian Balanean
}

Scientific Manager Nestle Infant Nutrition Romania

Dear Readers,

For healthy development and growth in childhood, the intestinal flora, or microbiome, plays a key role. The particular makeup of breast milk provides the optimum nutrition for the newly born child and a support for the microbiome. This contains an abundance of positive substances. Current interest has been focusing on the so-called human milk oligosaccharides (HMO) as a centre of particular attention, which represents an essential part of the content of breast milk. The importance of these components has already been known to researchers for some time, but it has not been possible until recently to enrich infant formulae with selected and particularly important HMOs. These HMOs are the same as those found in breast milk. They have been positively evaluated both in Europe and in the United States (,,Novel Food" status by the EFSA, GRAS status by the FDA). The first results of these studies look very promising. Learn more about this exciting topic on the following pages!

„An advance in research, as significant as the moon landing!" That is how otherwise very down-to-earth and serious experts enthuse about the most recent developments in food science. And not without good reason, because within just a short period of time the conditions have been created which open up a new era in infant nutrition. The secret is called HMO.

Ten years ago the microbiome - which used to be referred to as the intestinal flora - was a field of study which was only understood by a few specialists in microbiology. In the meantime, however, this has become a booming area of research. It is becoming increasingly evident that this extraordinarily complex area can be attributed a decisive role in development, well-being and longterm health. Even feelings and behaviour seem to be influenced by it. The microbiome is increasingly being recognised as an organ in its own right - a quite special organ, as it changes throughout the course of a lifetime - and we can change it, as well!

\section{Millions of good friends}

The dimensions alone are impressive! Because the microbiome includes bacteria, fungi, viruses, and the metabolites produced by them in the body. The intestinal microbiome - i.e. all of the microbes that inhabit the human gut, give rise to some amazing numbers:

Intestinal flora of adults:

- 100 trillion $\left(10^{14}\right)$ bacteria

- $10 \mathrm{x}$ more than all cells in the body

- About 36,000 different species

- Approx. 1-2 kg of biomass

- Intestinal microbiome encompasses about 3.3 million genes

- Roughly 150 times more than the human genome

This microbiome obviously does not exist in a random form, there are preferred combinations of bacteria, the so-called enterotypes, which are apparently related to dietary habits.

\section{Is the gut a second brain?}

The relationship to health is particularly interesting. Because more and more diseases, which are not causally related to the intestines, seem to be significantly influenced by the state of the microbiome, including respiratory diseases, obesity, type 2 diabetes, allergies, and asthma. There is even evidence that autism, depression or chronic pain are due to disorders of the gut-brain axis. As a consequence of these new findings, the current Rome IV guidelines for functional gastro-intestinal disorders (FGID) recommend in future to use the wording "Disorders of Gut-Brain Interaction" (DGBI).

Some are even now saying that the intestine is a "second brain", which controls our thoughts and (sub)conscious similarly to the brain. It is therefore 
an inescapable conclusion that a well balanced intestinal microbiome has a significant influence on health, and therefore nutrition is an important factor (Fig. 1).

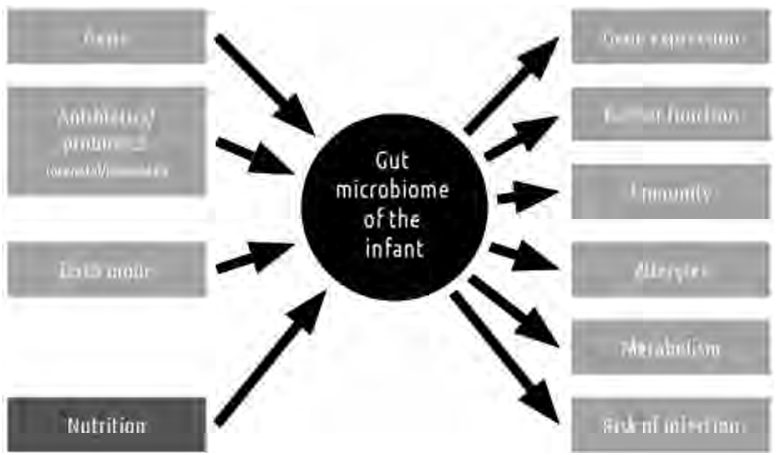

FIGURE 1. Control centre gut microbiome Modified from Houghteling et al. J Pediatr Gastroenterol Nutr.2015; 60(3):294-307

\section{Breast milk is the best}

In this context, the composition of breast milk plays a decisive role. Because in this way, the infantile organism is introduced to the most important components of a healthy intestinal flora. Breast milk provides the most important ,probiotic“ bifidus and lactobacillus bacteria, whose positive effect has been well-known for a long time.

With $800 \mathrm{ml}$ breast milk per day, a baby ingests about 1 million bacteria, which includes bifidus and lactobacillus bacteria. Many factors contribute to the bifidogenic effect of breast milk.

\section{All-round talent HMO}

Amongst the many components with a protective effect, the human milk oligosaccharides (HMOs) play a decisive role. HMOs occur solely in breast milk. After the milk sugar lactose and the milk fats, milk oligosaccharides represent the third largest component group in breast milk (Fig. 2).

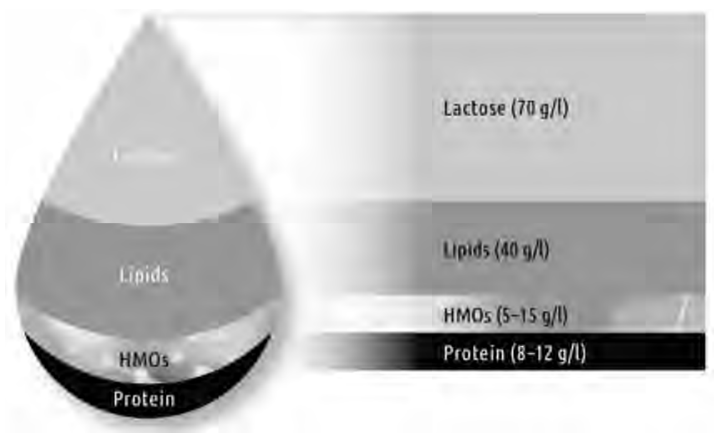

FIGURE 2. Third largest solid component in breast milk Jantscher-Krenn et al. Minerva Pediatr 2012a;64:83-89
HMOs occur in a great diversity of different structures and amounts. The individual composition depends upon various factors. The quantitative largest compound is in this case 2'-fucosyllactose (2'FL), which accounts in most cases for almost one third of the entire HMO content. (Fig. 3). 2'FL has a particularly positive effect on the microbiome of breastfed children.

For more than one hundred years, scientists and paediatricians have been fascinated about these important components in breast milk, both from their diversity as well as from their benefit on the infantile organism. More recently, technological advances in the manufacture of individual HMOs have made it possible to study these also in placebo-controlled and randomised clinical trials.

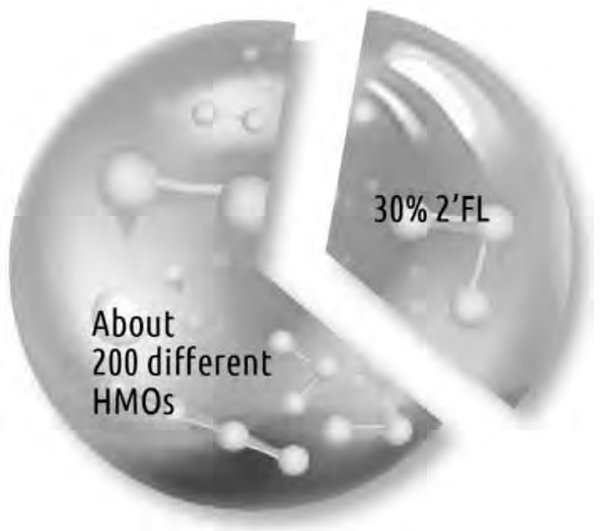

FIGURE 3. Largest proportion of the HMOs:2'FL Kobata A, Proc Jpn Acad Ser B Phys Biol Sci, 2010

\section{Complex sugars with effect}

Human milk oligosaccharides (HMOs) are an essential nutritional component for breastfed children. A lack of these components is the most important difference between breast milk and infant formula.

Human milk oligosaccharides have a special structure, they occur in a large variety of forms and make up the third largest component in breast milk. HMOs are relatively simple in their structure, but ultimately all of them are based on five basic substances. Almost all HMOs are based on lactose, which is modified in the mammary gland by the deposition of monosaccharides such as fucose, $\mathrm{N}$ acetylglucosamine, and/or sialic acid. In this way, complex structures with very specific links are formed, which is the basis for the multi-functionality of HMO (Fig. 4). 


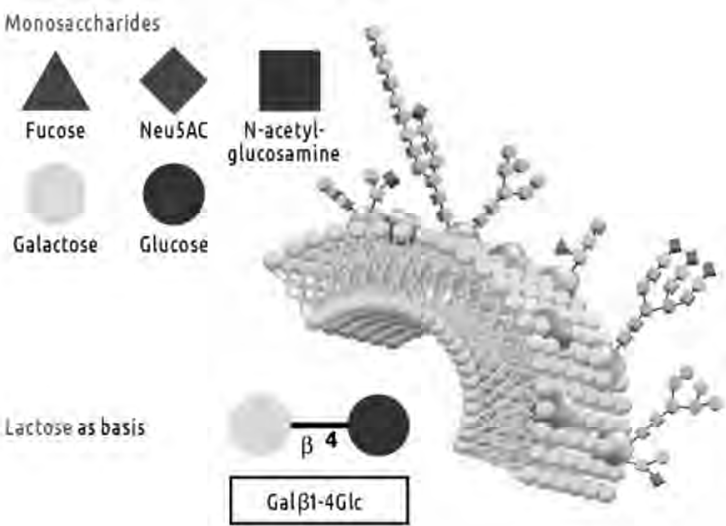

FIGURE 4. Same building blocks, different links Adapted from: Rohrer J: HPAE-PAD Peak Area Response of Glycoprotein Oligosaccharides, Thermo Fisher Scientific Tech Note 133, 2016

The total concentration of HMOs in mature breast milk is between 10 and $15 \mathrm{~g} / \mathrm{L}$ - in colostrum this may be even higher. For most mothers, 2'FL makes up the largest proportion with about 30 percent.

\section{Bifidogenic effect}

If the microbiotic composition of the gut of breastfed infants is analysed, normally there is a distinct prevalence of bifidus bacteria and a smaller proportion of potentially pathogenic bacteria. By stimulation of bifidus bacteria using different HMOs, the introduction of pathogenic substances can be prevented, as György was able to establish already in 1955. More recent data allow the conclusion to be made that apparently certain bacterial sub-strains possess the ability to utilise the HMOs and/or different forms of HMOs (Fig. 5).

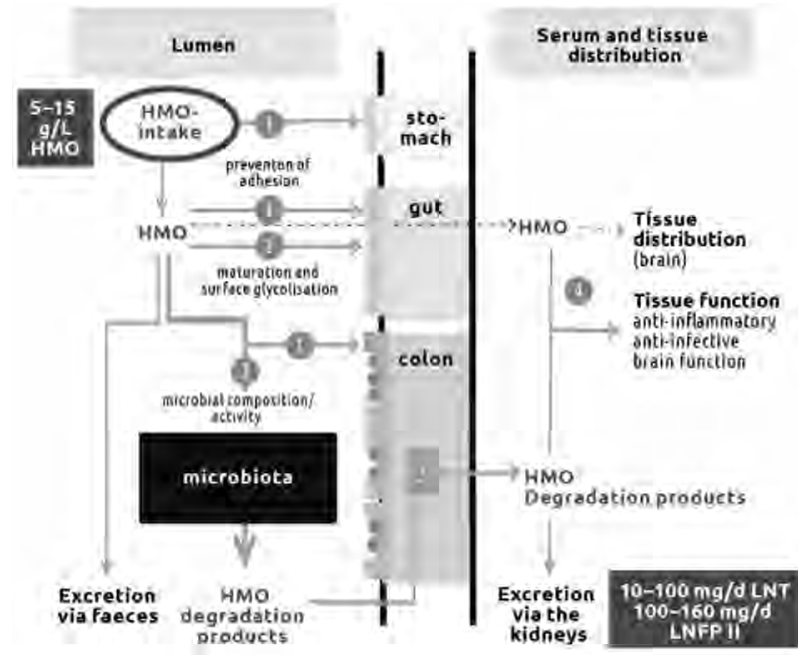

FIGURE 5. Intake, metabolism and potential functions Kunz C, Das Nest 40, 2016
In this way, it becomes progressively clearer that HMOs are really great all-rounders for a healthy development (Fig. 6).

- HMOs promote the growth of specific bifdus bacteria in the infantile gut

- HMOs are the "decoy" for pathogens. They adhere to the HMOs and are excreted together with them in the stools

- HMOs strengthen the gut barrier function, they prevent adhesion of potential pathogens to the intestinal cells by "reprogramming" them

- HMOs have an immune regulatory effect, they contribute to the development of balanced Th1/Th2-immune responses

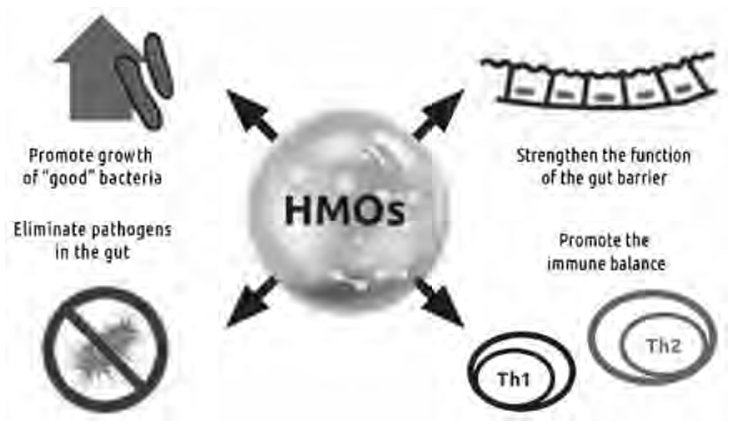

FIGURE 6. Key mechanisms of HMOs

Jantscher-Krenn, Bode: Minerva Paediatr. 2012; Bode L, Glycobiology, 2012;

Smilowitz JT, Lebrilla CB, Mills DA et al. Annu Rev Nutr. 2014

\section{HMO: More than just prebiotics}

In more recent years, it has become normal practice to add certain carbohydrates such as galacto- (GOS) and fructooligosaccharides (FOS) to infant formula, as they promise to have a positive effect on the development of the infantile microbiome. They are not the same as human oligosaccharides (HMOs), however. These have in their mechanism of action a more far reaching action than GOS/FOS, however.

A significant difference is to be found in that FOS does not occur in breast milk at all and GOS is only present in extremely low amounts. Although these prebiotics also promote the colonisation of bifidogenic bacteria in the gut of neonates and small children, their effect is altogether considerably more limited than in the case of HMOs. This is due to the differences in structure:

HMOs have more complex structures and components, which do not arise in FOS/GOS.

- FOS have two components and a kind of link as simple as Lego building blocks

- GOS also have two components, however, different forms of linkage 
- HMOs consist of at least three components and have a multitude of different linkages

Many functions of HMOs are dependent upon structure. For this reason, FOS/GOS are not able to achieve the mechanism of action of the HMOs.

\section{Prebiotics}

- Have a less specific action on the gut microbiome

- Are not able to "lure" and eliminate pathogens

- Are not able to directly strengthen the gut barrier function

- Have no direct effect on the immune balance

First HMOs offer the positive characteristics of breast milk and make it possible to offer a comprehensive protective effect to the infantile flora of the gut.

\section{On the trail of the oligosaccharides}

The first description of micro-organisms such as lactobacillus and bifidobacteria was already made around the year 1900 . Even at that time, there were the first indications that the different composition of faeces of breastfed and bottle-fed children was associated with the different carbohydrates. Then at the beginning of the 1930's, the first individual breast milk oligosaccharides were characterised.

A quantum leap in HMO research was achieved in the 1950's when the paediatrician Paul György and the chemist Richard Kuhn were able to demon- strate that the growth factor for Lactobacillus bifidus (later classified as Bifidobacterium bifidus) exists in breast milk as oligosaccharides.

In the following years, it was possible to discover and classify more than a dozen HMOs. Since the turn of the millennium, impressive advances have been made in the development of methods for analysing the structure in detail, even with extremely low amounts of milk. At the same time, it was also possible to produce larger amounts of individual HMOs using chemical and biotechnical means.

After the prebiotic effect had been initially investigated, the focus was also increasingly put on other health advantages which are associated with HMOs. It was now discussed how and which oligosaccharides could be added to infant's milk in order to achieve similar positive effects such as HMOs in breast milk.

In 2012, the first clinical trial was carried out with HMO supplemented nutrition, after numerous in-vitro and animal studies had shown very promising results.

\section{Important abbreviations}

$\mathrm{HMO}=$ Human Milk Oligosaccharides

2' $\mathrm{FL}=2$ ' - Fucosyllactose

LNT $=$ Lacto-N-tetraose

GOS $=$ Galactooligosaccharides

FOS $=$ Fructooligosaccharides

FGID $=$ Functional Gastro-intestinal disorders

DGBI $=$ disorders of Gut-Brain interaction

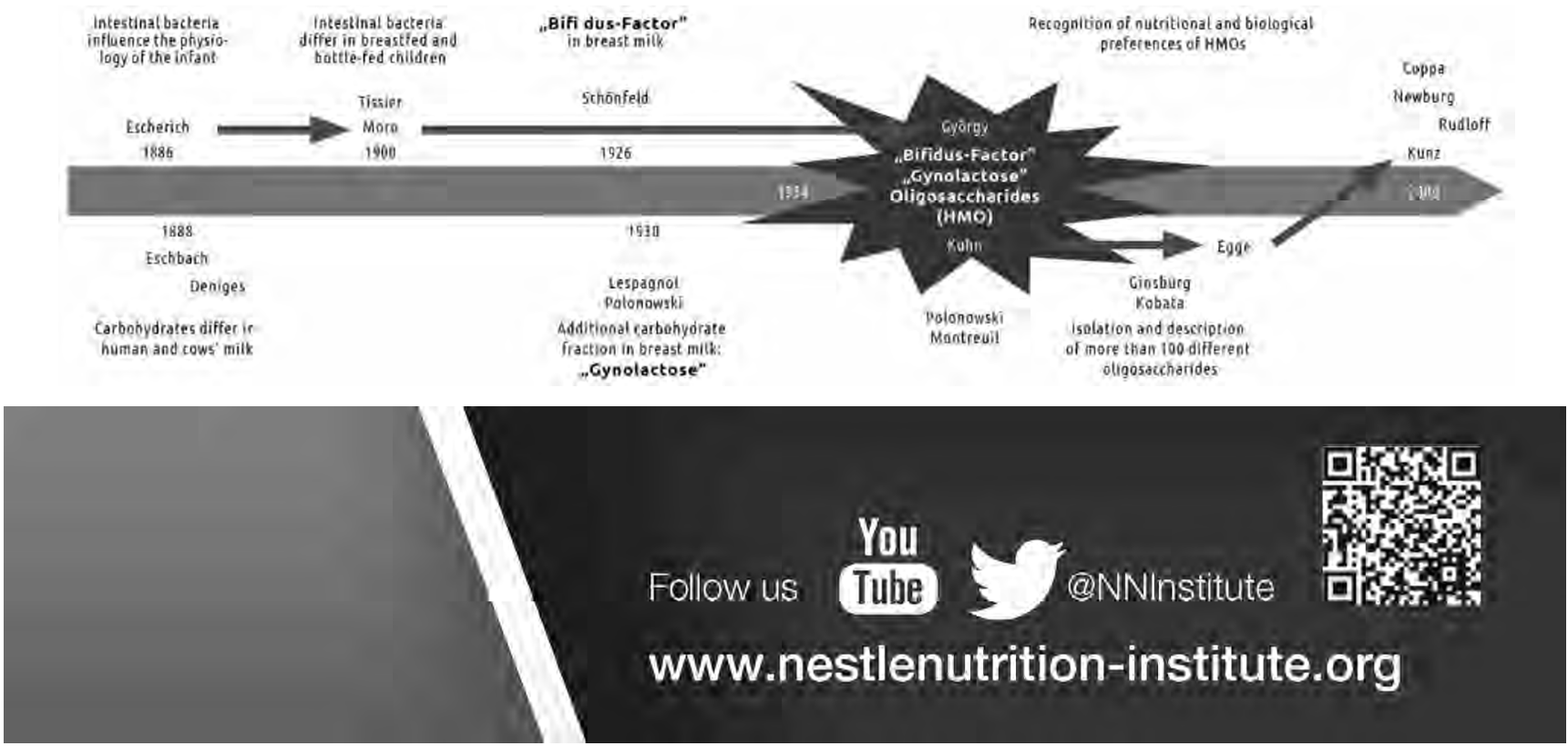

